\title{
Explainable analytics: Understanding causes, correcting errors, and increasingly achieving perfect accuracy from nature of distinguishable patterns
}

Hao-Ting Pai ( $\sim$ greatpai@hotmail.com )

National Yunlin University of Science and Technology

Chung-Chian Hsu

National Yunlin University of Science and Technology

\section{Research Article}

Keywords:

Posted Date: December 22nd, 2021

DOI: https://doi.org/10.21203/rs.3.rs-1130288/v2

License: (c) (i) This work is licensed under a Creative Commons Attribution 4.0 International License.

Read Full License 


\section{Abstract}

In addition to pursue accurate analytics, it is invaluable to clarify how and why inaccuracy exists. We propose a transparent classification method (TC). In training, we discover patterns from positive and negative observations respectively; next, patterns are excluded if they appear in both types. In testing, observations are scored by the pure patterns and connected like social networks. Based on set theory, pure patterns have explanatory power for distinguishing tangled relationship between negative and positive observations. Experimental results demonstrate that TC can identify all positive (e.g., malignant) observations at low ratios of training to testing, e.g., 1:9 in Breast Cancer Wisconsin (Original) and 3:7 in Contraceptive Method Choice dataset. Without fine-tuned parameters and random selection, TC eliminates uncertainty of the methodology. TC can visualize causes, and therefore, prediction errors are traceable and can be corrected. Further, TC shows potential of identifying whether the ground truth is incorrect (e.g., diagnostic errors).

\section{Main Text}

Accurate prediction plays a pivotal role in analytics; however, in reality people usually face the challenge of explaining how and why the prediction is inaccurate ${ }^{1-2}$. By the survey ${ }^{3}$, outpatient diagnostic errors are at a rate of $5.08 \%$ (around 12 million US adults) per year. Even reducing only $1 \%$ errors, the lives of million people are saved. We consider three major issues of errors. First, fault in data, human mistakes or defective machines can produce faulty data. Without domain knowledge, such a fault is difficult to correct. Nevertheless, we should remove inconsistency, i.e., observations of positive class are identical to those of negative one. In addition, positive and negative observations may have similar patterns that are inextricably interwoven, e.g., people with similar profiles may exhibit different behavior. Lim et al. ${ }^{4}$ show the contraceptive method choice (CMC) dataset ${ }^{5}$ is the most difficult to classify, and especially the minimum error rates are greater than 0.4 . Second, mismatches between data and methods, data contains categorical (e.g., country), numerical (e.g., age), or both types, which have natural constraints on analysis. For categorical values, only the number of items and the mode are statistically relevant ${ }^{6}$ so that a numerical-orientated method is inherently inadequate. For numerical values, they can be transformed into categorical ones by discretization ${ }^{7}$, which have been widely applied to knowledge discovery and data mining (KDD) applications ${ }^{8}$. However, bias occurs if categories are unrepresentative of numerical values. Third, big data challenge, the complexity of data is determined by the number of rows and features (columns). Particularly, computation tasks increase rapidly with the number of features, as known curse of dimensionality $(\mathrm{CoD})^{9}$. To cope with $\mathrm{CoD}$, dimension reduction and feature selection methods reduce the complexity by extracting information that is practical for classification and cluster analysis. The extraction, a trade-off between efficiency and effectiveness, may involve pruning large amounts of data. Actually, such a pruning manner may have pitfalls ${ }^{10}$ or miss clues to errors.

\section{Results}


We conducted experiments with two datasets: Breast Cancer Wisconsin (Original) (BCWO) and Contraceptive Method Choice $(C M C)^{5}$. Figure $2(A)(B)$ and $(C)(D)$ show the results of BCWO and CMC respectively. In Figure $2(A)$, TC achieves perfect recall (i.e., recall=1.0) at the lowest ratio (i.e., 1:9) and 7 other ratios. It means TC is not only accurate in small amounts of data but also stable when data increases. TC has one error at the ratios $2: 8$ and 3:7 because the positive observation $P O_{223}$ is predicted as a negative one. Since the ratio $4: 6, P_{223}$ belongs to training data and is not used in testing. For further exploration, at the ratio $10: 10^{\#}, P O_{223}$ is irrelevant to PPP except itself. Indeed, $P O_{223}$ is related to PP that are also relevant to NO. PPP can eliminate impingement of $P_{223}$ on other observations. Novel observations are regarded as positive ones if they are unrelated to both PPP and PNP. Some of NO are predicted as positive so that TC gets lower performance in terms of PR. With increased training observations, TC may obtain sufficient PP or NP to provide accurate results and their causes, e.g., at the ratio $1: 9, P O_{627}$ has a novelty score of 70 by rule 3 ; at the ratio $2: 8, P O_{627}$ contains 3 patterns in PPP, namely, ['8|0.8'], ['6|1.5', '8|0.8'], and ['1|0.9', '8|0.8'] and has a positive score of 16 by rule 1. Figure 2 (B) shows the suitable granularity of discretization gives broad vision for discovering more clues. At the ratio 1:9 TC has one error, which is also caused by $P O_{223}$. At the ratio $2: 8, P O_{223}$ has 1 pattern ['3|0.0', '5|0.0'] in PPP and is related to $P O_{33}$ and $P O_{102}$. At the ratio $3: 7, P O_{223}$ has 1 pattern ['3|0.0', '5|0.0'] in PPP and related to $P O_{33}, P O_{102}$, and $P O_{143}$. Since the ratio 4:6, $P O_{223}$ belongs to training data and is not used in testing. In Figure 2 (C) (D) at the ratios 1:9 and 2:8, RE and PR are zero due to none of negative observations in training (TRN). At the ratio 3:7, insufficient TRN leads to extremes as perfect RE but relatively low PR. The error rate is less than 0.4 at the ratio 7:3. In CMC, profile data is incapable of explaining behavior because (D) indicates 33\% (i.e., (1473-980)/1473) cases are inconsistent inherently. In Figure 3, we provide visualization of TC. Compared to images, analysis of categorical and numerical data was difficult to visualize how causes are related to results.

\section{Discussion}

Figure $3(\mathrm{~A})$ shows TC successfully predicts $O_{104}$ is positive because $O_{104}$ is related to the six pure positive patterns that are obtained from respective positive observations of training. Moreover, the thickness of lines represents the score as well as the positive degree. Figure 3 (B) presents the association between $\mathrm{O}_{104}$ and others. In the $\mathrm{O}_{104}$-headed group, the most common positive pattern is '0|1.3', and $O_{24}$ and $O_{57}$ have significant influence on $O_{104}$. Figure $4(\mathrm{~A})(\mathrm{B})(\mathrm{C})$ illustrate TC is tolerant of faulty class labels (ground truth) in terms of testing, training, and both cases. Figure 4 (D) demonstrates TC can correct errors in class labels. Specifically, observations of faulty class labels have extreme values of PS or NS, which becomes significant with increasing data. Based on profound knowledge of most cases, TC would be useful for checking whether the original judgement (e.g., diagnosis) deserves further inspection.

\section{Methods}


We propose a method of transparent classification, named TC, which not only pursues accuracy but also clarifies cause of inaccuracy. Further, the design principles of TC ensure reproducibility ${ }^{11}$. Figure 1 shows processes of TC. In data preprocessing, TC handles missing values and mixed values. Without involving randomness and reduction, TC delivers intrinsic nature of data. In identifying distinguishable patterns, TC finds patterns from training observations, which are used for predicting which class a test observation belongs to, e.g., a malignant or benign tumor. Through increasing ratios of training to testing, TC represents the forest and the trees, and input data is given in sequence. To avoid CoD, TC finds patterns by intersecting pairwise observations in each of the classes, which possess essential features of data in miniature. In the worst case of TC, $n$ observations produce only patterns. By contrast, KDD faces the challenge of $\mathrm{CoD}$, i.e., given the lowest threshold, $k$ items yield $2^{k}$ itemsets $^{12}$, and large amounts of itemsets are pruned if the threshold is high. In positive patterns (PP), TC obtains PP from positive training observations (PO). For pure PP (PPP), TC excludes any positive pattern that also appears in negative training observations (NO). By set theory, the exclusion implies none of PPP is included in any of NO and hence TC can distinguish between PO and NO. Analogously, Negative patterns (NP) and pure NP(PNP) are the counterpart of PP and PPP. Without involving either fine-tuned parameters or random selection, TC eliminates uncertainty of the methodology. In establishing the causes, TC accumulates positive, negative, and novel degrees of a test observation $O_{t}$ by rule 1,2 , and 3 which associate patterns with the observation and provide obvious clues for judgement. In rule $1, O_{t}$ containing patterns in PPP gets a positive score (PS). In rule $2, O_{t}$ containing patterns in PNP gets a negative score (NS). In rule $3, O_{t}$ containing none of patterns in PPP and PNP is considered novel and gets a novelty score (NT) equal to the number of training observations. In understanding results of analytics, we evaluate performance of TC by three measures, Precision, Recall, and AUC (Area under Curve) ${ }^{8,12}$. According to the standard of diagnostic medicine ${ }^{13}$ : $A U C=0.5$, no discrimination; $0.7 \leq A U C<0.8$, acceptable; $0.8 \leq A U C<0.9$, excellent; and $0.9 \leq \mathrm{AUC} \leq 1$, outstanding. In cause for prediction errors, error 1 (false positive ${ }^{14}$ ) occurs if $O_{t}$ is predicted as positive but actually negative, denoted by $N O_{t^{*}}$. By cause $1.1, N O_{t^{*}}$ contains pure positive patterns although it should not. By cause 1.2, $N O_{t^{\star}}$ is novel, namely, containing no pattern in PPP and PNP. Error 2 (false negative ${ }^{14}$ ) occurs if $O_{t}$ is predicted as negative but actually positive, denoted by $P O_{t^{*}}$. By cause 2.1, $P O_{t^{\star}}$ contains pure negative patterns but no pure positive pattern although it should. Prediction errors occur due to insufficient training data or labelling errors in training data. Increasing training data helps to reduce prediction errors. If the portion of labelling errors is small, TC has the potential of identifying labelling errors. Specifically, false negatives usually have a small NS.

\section{Declarations}

Acknowledgments: We thank the editor and reviewers for offering comments on the manuscript.

Funding: This work was supported by the Ministry of Science and Technology (MOST) in Taiwan. 
MOST (grant no. 110-2410-H-224-037-): H.T.P.

MOST (grant no. 110-2410-H-224-009): C.C.H.

\section{Author contributions:}

Conceptualization: H.T.P. conceived the idea and C.C.H. provided for guidance.

Methodology: H.T.P.

Experiments: H.T.P.

Visualization: H.T.P.

Funding acquisition: H.T.P., C.C.H.

Writing - original draft: H.T.P.

Writing - review \& editing: H.T.P., C.C.H.

Competing interests: Authors declare that they have no competing interests.

\section{References}

1. Description prediction, explanation. Nat Hum Behav, 5, 1261 (2021).

2. Gunning, D. et al. XAI-Explainable artificial intelligence. Sci. Robot, 4, eaay7120 (2019).

3. Singh, H., Meyer, A. N. \& Thomas, E. J. The frequency of diagnostic errors in outpatient care: estimations from three large observational studies involving US adult populations. BMJ Qual. Saf, 23, 727-731 (2014).

4. Lim, T. S., Loh, W. Y. \& Shih, Y. S. A comparison of prediction accuracy, complexity, and training time of thirty-three old and new classification algorithms. Mach. Learn, 40, 203-228 (2000).

5. Dheeru, D., Graff, C. \& UCI Machine Learning Repository. (2019); http://archive.ics.uci.edu/ml

6. Stevens, S. S. On the theory of scales of measurement. Science, 103, 677-680 (1946).

7. Garcia, S., Luengo, J., Sáez, J. A., Lopez, V. \& Herrera, F. A survey of discretization techniques:

Taxonomy and empirical analysis in supervised learning. IEEE Trans. Knowl. Data Eng, 25, 734-750 (2012).

8. Tan, P. N., Steinbach, M. \& Kumar, V. Introduction to Data Mining (Pearson, 2020).

9. Altman, N. \& Krzywinski, M. The curse(s) of dimensionality. Nat Methods, 15, 399-400 (2018).

10. Jain, A. \& Zongker, D. Feature selection: Evaluation, application, and small sample performance. IEEE Trans. Pattern Anal. Mach. Intell, 19, 153-158 (1997).

11. McNutt, M.Reproducibility. Science.343,229(2014) 
12. Zaki, M. J. \& Meira, W. Jr Data Mining and Machine Learning: Fundamental Concepts and Algorithms (Cambridge University Press, 2020).

13. Mandrekar, J. N. Receiver operating characteristic curve in diagnostic test assessment. J. Thorac. Oncol, 5, 1315-1316 (2010).

14. National Cancer Institute (2021); https://www.cancer.gov/publications/dictionaries/cancerterms/def/false-positive-test-result

\section{Figures}

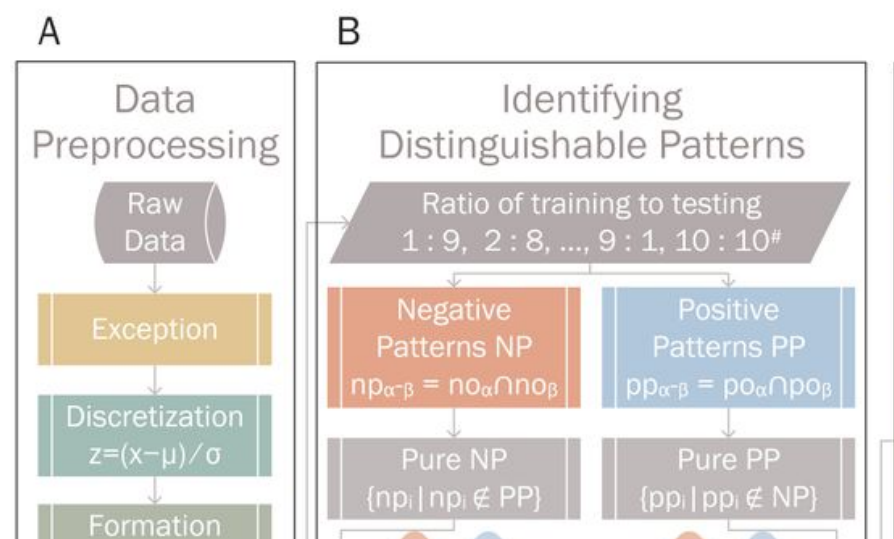

\begin{tabular}{|c|}
\hline $\begin{array}{l}\text { Establishing } \\
\text { the Causes }\end{array}$ \\
\hline $\begin{array}{l}\quad \text { Classification by scoring } \\
\text { Rule } 1 . \\
\text { If } p p p \subseteq \mathrm{O}_{\mathrm{t}} \text {, } \\
\mathrm{PS}_{\mathrm{t}}=\mathrm{PS}_{\mathrm{t}}+|\mathrm{PO}(\mathrm{ppp})|^{*}|p p p| \\
\text { Rule } 2 . \\
\text { If pnp } \subseteq \mathrm{O}_{\mathrm{t}}, \\
\mathrm{NS}_{\mathrm{t}}=\mathrm{NS}_{\mathrm{t}}+\mid \mathrm{NO} \text { (pnp) }|*| p n p \mid \\
\text { Rule } 3 . \\
\nexists i, j, p p p_{i} \subseteq \mathrm{O}_{\mathrm{t}}, p^{*} p_{j} \subseteq \mathrm{O}_{\mathrm{t}} \text {, then }\end{array}$ \\
\hline
\end{tabular}

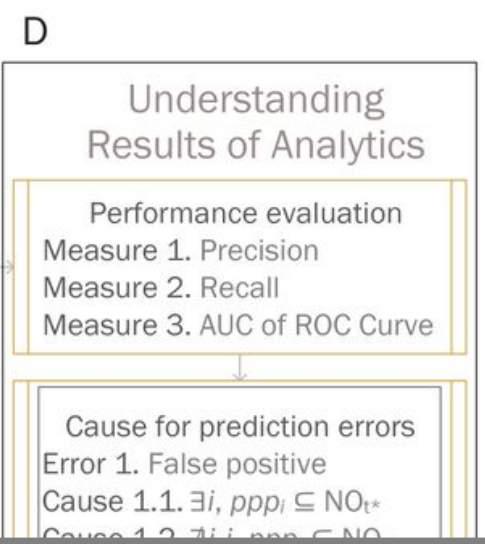

\section{Figure 1}

Processes of the transparent classification. (A) Exception treats missing values as categorical values instead of guesses. Discretization transfers numerical values to categorical ones by using z-score, where $x$ are numerical values of a feature, $\mu$ is the mean and $\sigma$ is the standard deviation. Formation defines the relations between features $f$ and categorical values e. Consistency removes the contradictory observations that have identical features but different class labels. (B) Ratio of training to testing divides data into two parts: training and testing. In training, observations are split positive observations $\mathrm{PO}$ and negative observations NO. A positive pattern $p p_{\alpha-\beta}$ was discovered by intersecting $p o_{a}$ and $p o_{\beta}$, where $\alpha=1$, $2, \ldots, n$ and $\beta=a, a+1, \ldots, n$, e.g., given $n=3$, then $p p_{1-1}=p o_{1} \cap p o_{1}=\left\{f_{1}\left|e_{1,1}, f_{2}\right| e_{2,2}, f_{3} \mid e_{3,1}\right\}, p p_{1-2}=p o_{1} \cap p o_{2}=$ $\left\{f_{1}\left|e_{1,1}, f_{2}\right| e_{2,2}, f_{3} \mid e_{3,1}\right\} \cap\left\{f_{1}\left|e_{1,1}, f_{2}\right| e_{2,1}, f_{3} \mid e_{3,1}\right\}=\left\{f_{1}\left|e_{1,1}, f_{3}\right| e_{3,1}\right\}, p p_{1-3}=p o_{1} \cap p o_{3}, p p_{2-2}=p o_{2}, p p_{2-3}=p o_{2} \cap p o_{3}$, and $p p_{3-3}=p o_{3}$. Note positive observations themselves are positive patterns, e.g., $p p_{1-1}$. A negative pattern $n p_{a-\beta}$ was found by $n o_{a} \cap n o_{\beta}$, and negative observations themselves are negative patterns. Pure PP (PPP) excludes $p p_{a-\beta}$ that appears in any negative observation. Pure NP(PNP) excludes $n p_{a-\beta}$ that appears in any positive observation. (C) In testing of an observation $O_{t}$, classification by scoring produces five 
outputs: PS, NS, NT, PSP, and PSO. PS stores observation's positive score by Rule 1: $O_{t}$ contains a pure positive pattern $p p p$, increase $P S$ by the number of features in $p p p$, multiplied by the number of positive observations containing $p p p$. Rule 2: if $O_{t}$ contains a pure negative pattern $p n p$, increase $N S$ by the number of features in $p n p$ multiplied by the number of negative observations containing pnp. Rule 3 : if $O_{t}$ does not contain any $p p p$ and $p n p$, assign $N T$ to the number of training observations. $P S P$ stores $p p p_{a-\beta}$ related to $O_{t}$. PSO stores the training observations which contain $p p p_{\alpha-\beta}$. If $P S$ or $N T$ is greater than 0 , classify $O_{t}$ as positive otherwise negative. (D) Performance evaluation demonstrates the accuracy of TC. Cause for prediction errors, based on set theory, provides rational explanations for errors caused by TC. 


A
\begin{tabular}{|r|l|l|l|l|r|r|r|r|r|r|r|r|r|r|}
\hline RA & RE & PR & AU & ER & TP & TN & FP & FN & TRP & TRN & TEP & TEN & UPP & PPP \\
\hline $1: 9$ & 1.000 & 0.701 & 0.905 & 0.140 & 206 & 335 & 88 & 0 & 35 & 35 & 206 & 423 & 251 & 201 \\
\hline $2: 8$ & 0.994 & 0.777 & 0.976 & 0.093 & 178 & 329 & 51 & 1 & 62 & 78 & 179 & 380 & 592 & 468 \\
\hline $3: 7$ & 0.993 & 0.760 & 0.988 & 0.100 & 152 & 288 & 48 & 1 & 88 & 122 & 153 & 336 & 1007 & 845 \\
\hline $4: 6$ & 1.000 & 0.777 & 0.996 & 0.079 & 115 & 271 & 33 & 0 & 126 & 154 & 115 & 304 & 1630 & 1364 \\
\hline $5: 5$ & 1.000 & 0.752 & 0.997 & 0.077 & 82 & 240 & 27 & 0 & 159 & 191 & 82 & 267 & 2266 & 1880 \\
\hline $6: 4$ & 1.000 & 0.805 & 0.997 & 0.057 & 66 & 198 & 16 & 0 & 175 & 244 & 66 & 214 & 2599 & 2163 \\
\hline $7: 3$ & 1.000 & 0.810 & 0.996 & 0.052 & 47 & 152 & 11 & 0 & 194 & 295 & 47 & 163 & 2968 & 2493 \\
\hline $8: 2$ & 1.000 & 0.833 & 0.998 & 0.050 & 35 & 98 & 7 & 0 & 206 & 353 & 35 & 105 & 3225 & 2710 \\
\hline $9: 1$ & 1.000 & 0.765 & 0.999 & 0.057 & 13 & 53 & 4 & 0 & 228 & 401 & 13 & 57 & 3723 & 3162 \\
\hline $10: 10^{*}$ & 1.000 & 1.000 & 1.000 & 0.000 & 241 & 458 & 0 & 0 & 241 & 458 & 241 & 458 & 4023 & 3422 \\
\hline
\end{tabular}
RA: Ratios of training to testing; RE: Recall; PR: Precision; AU: AUC of ROC Curve; ER: Error rate; TP: True
positive; TN: True negative; FN: False negative; FP: False positive; TRP: Positive observations of training; TRN:
Negative observations of training; TEP: Positive observations of testing; TEN: Negative observations of testing;
UPP: Unique positive patterns; PPP: Pure positive patterns. In RA, for comprehensive exploration, 10:10 means
we respectively give the entire data in training and test.
?

B

\begin{tabular}{|r|l|l|l|l|r|r|r|r|r|r|r|r|r|r|}
\hline RA & RE & PR & AU & ER & TP & TN & FP & FN & TRP & TRN & TEP & TEN & UPP & PPP \\
\hline $1: 9$ & 0.995 & 0.670 & 0.904 & 0.162 & 205 & 322 & 101 & 1 & 35 & 35 & 206 & 423 & 359 & 273 \\
\hline $2: 8$ & 1.000 & 0.731 & 0.975 & 0.118 & 179 & 314 & 66 & 0 & 62 & 78 & 179 & 380 & 861 & 677 \\
\hline $3: 7$ & 1.000 & 0.750 & 0.986 & 0.104 & 153 & 285 & 51 & 0 & 88 & 122 & 153 & 336 & 1390 & 1153 \\
\hline $4: 6$ & 1.000 & 0.737 & 0.992 & 0.098 & 115 & 263 & 41 & 0 & 126 & 154 & 115 & 304 & 2308 & 1889 \\
\hline $5: 5$ & 1.000 & 0.766 & 0.997 & 0.072 & 82 & 242 & 25 & 0 & 159 & 191 & 82 & 267 & 3211 & 2564 \\
\hline $6: 4$ & 1.000 & 0.815 & 0.998 & 0.054 & 66 & 199 & 15 & 0 & 175 & 244 & 66 & 214 & 3666 & 2959 \\
\hline $7: 3$ & 1.000 & 0.839 & 0.998 & 0.043 & 47 & 154 & 9 & 0 & 194 & 295 & 47 & 163 & 4162 & 3371 \\
\hline $8: 2$ & 1.000 & 0.875 & 0.998 & 0.036 & 35 & 100 & 5 & 0 & 206 & 353 & 35 & 105 & 4555 & 3697 \\
\hline $9: 1$ & 1.000 & 0.765 & 1.000 & 0.057 & 13 & 53 & 4 & 0 & 228 & 401 & 13 & 57 & 5286 & 4350 \\
\hline $10: 10^{\sharp}$ & 1.000 & 1.000 & 1.000 & 0.000 & 241 & 458 & 0 & 0 & 241 & 458 & 241 & 458 & 5675 & 4687 \\
\hline
\end{tabular}

C

\begin{tabular}{|r|l|l|l|l|r|r|r|r|r|r|r|r|r|r|}
\hline RA & RE & PR & AU & ER & TP & TN & FP & FN & TRP & TRN & TEP & TEN & UPP & PPP \\
\hline $1: 9$ & 1.000 & 0.362 & 0.432 & 0.638 & 456 & 0 & 803 & 0 & 140 & 0 & 456 & 803 & 1539 & 1539 \\
\hline $2: 8$ & 1.000 & 0.282 & 0.428 & 0.718 & 316 & 0 & 803 & 0 & 280 & 0 & 316 & 803 & 3555 & 3555 \\
\hline $3: 7$ & 1.000 & 0.203 & 0.566 & 0.795 & 198 & 3 & 778 & 0 & 398 & 22 & 198 & 781 & 5539 & 4252 \\
\hline $4: 6$ & 0.944 & 0.259 & 0.662 & 0.652 & 187 & 105 & 536 & 11 & 398 & 162 & 198 & 641 & 5539 & 2762 \\
\hline $5: 5$ & 0.843 & 0.321 & 0.653 & 0.549 & 167 & 148 & 353 & 31 & 398 & 302 & 198 & 501 & 5539 & 2141 \\
\hline $6: 4$ & 0.763 & 0.442 & 0.676 & 0.425 & 151 & 171 & 191 & 47 & 398 & 441 & 198 & 362 & 5539 & 1798 \\
\hline $7: 3$ & 0.703 & 0.517 & 0.673 & 0.390 & 121 & 135 & 113 & 51 & 424 & 555 & 172 & 248 & 5938 & 1783 \\
\hline $8: 2$ & 0.844 & 0.163 & 0.727 & 0.514 & 27 & 109 & 139 & 5 & 564 & 555 & 32 & 248 & 8345 & 2693 \\
\hline $9: 1$ & 0.000 & 0.000 & 0.500 & 0.536 & 0 & 65 & 75 & 0 & 596 & 663 & 0 & 140 & 8744 & 2728 \\
\hline $10: 10^{4}$ & 1.000 & 1.000 & 1.000 & 0.000 & 596 & 803 & 0 & 0 & 596 & 803 & 596 & 803 & 8744 & 2566 \\
\hline
\end{tabular}

D

\begin{tabular}{|r|l|l|l|l|r|r|r|r|r|r|r|r|r|r|}
\hline RA & RE & PR & AU & ER & TP & TN & FP & FN & TRP & TRN & TEP & TEN & UPP & PPP \\
\hline $1: 9$ & 1.000 & 0.367 & 0.503 & 0.633 & 324 & 0 & 558 & 0 & 98 & 0 & 324 & 558 & 1480 & 1480 \\
\hline $2: 8$ & 1.000 & 0.288 & 0.515 & 0.712 & 226 & 0 & 558 & 0 & 196 & 0 & 226 & 558 & 3524 & 3524 \\
\hline $3: 7$ & 1.000 & 0.213 & 0.632 & 0.770 & 143 & 15 & 528 & 0 & 279 & 15 & 143 & 543 & 5580 & 4293 \\
\hline $4: 6$ & 0.986 & 0.283 & 0.712 & 0.611 & 141 & 88 & 357 & 2 & 279 & 113 & 143 & 445 & 5580 & 2723 \\
\hline $5: 5$ & 0.958 & 0.369 & 0.751 & 0.490 & 137 & 113 & 234 & 6 & 279 & 211 & 143 & 347 & 5580 & 1978 \\
\hline $6: 4$ & 0.888 & 0.498 & 0.779 & 0.367 & 127 & 121 & 128 & 16 & 279 & 309 & 143 & 249 & 5580 & 1564 \\
\hline $7: 3$ & 0.866 & 0.569 & 0.778 & 0.320 & 103 & 97 & 78 & 16 & 303 & 383 & 119 & 175 & 5994 & 1572 \\
\hline $8: 2$ & 0.857 & 0.165 & 0.798 & 0.480 & 18 & 84 & 91 & 3 & 401 & 383 & 21 & 175 & 8192 & 2355 \\
\hline $9: 1$ & 0.000 & 0.000 & 0.500 & 0.592 & 0 & 40 & 58 & 0 & 422 & 460 & 0 & 98 & 8560 & 2320 \\
\hline $10: 10^{*}$ & 1.000 & 1.000 & 1.000 & 0.000 & 422 & 558 & 0 & 0 & 422 & 558 & 422 & 558 & 8560 & 2120 \\
\hline
\end{tabular}

Figure 2

Performance of TC in distinguishing between observations. (A) (B) In Breast Cancer Wisconsin (Original) data set (BCWO), we map class values "malignant" to " 1 " and "benign" to " 0 ". In case (A), the granularity of discretization is to the first decimal place, e.g. 1.68 $\approx 1.6$, while in case (B) we take an integer for the granularity, e.g., 1.68 1 1. (C) (D) In Contraceptive Method Choice data set (CMC), we map class values "1=No-use" to "1", "2=Long-term" to "0", and "3=Short-term" to "0". For (C) and (D), we set the same 
granularity as that of $(A)$ and $(B)$, respectively. For consistency, we remove observations that have identical features but different class labels. The number of observations is thus reduced from 1473 to 1399 in (C) and from 1473 to 980 in (D).

A

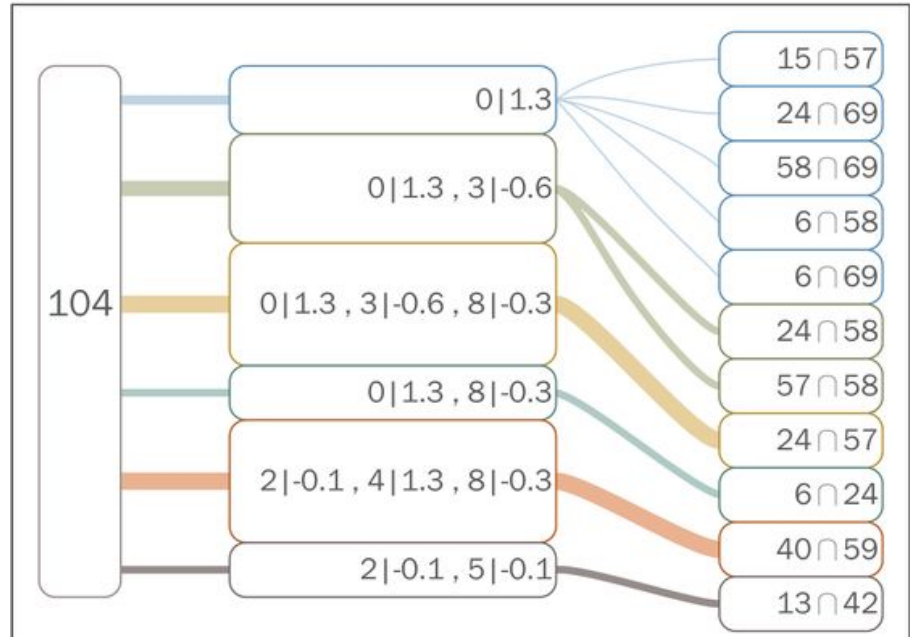

B

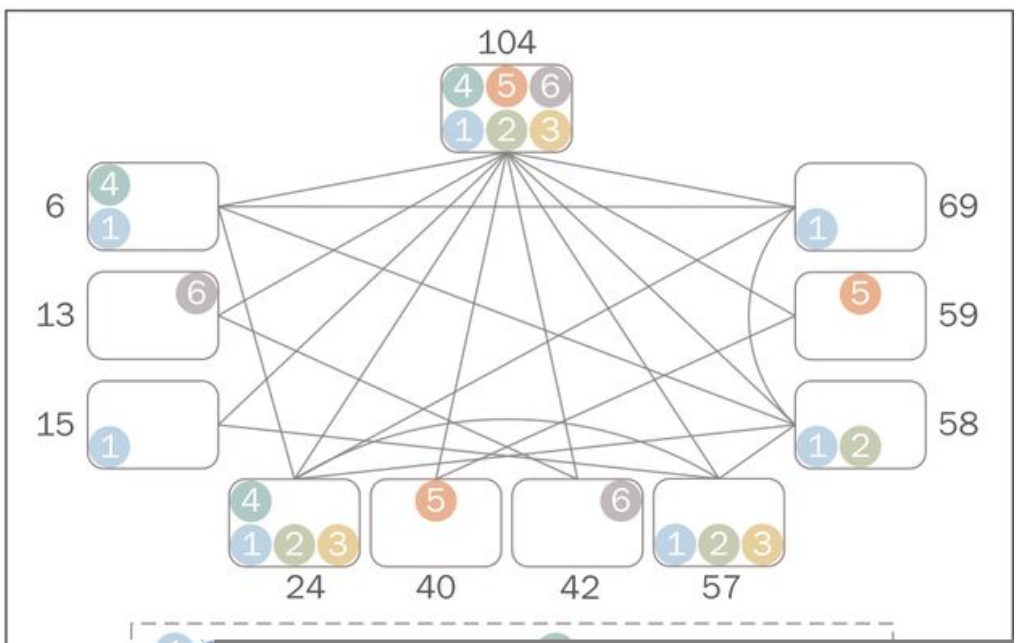

Figure 3

Association between patterns and observations in $\mathrm{BCWO}(\mathrm{A})$ at the ratio 1:9. 


A
\begin{tabular}{|r|l|l|l|l|l|r|r|r|r|r|r|r|r|r|}
\hline RA & RE & PR & AU & ER & \multicolumn{1}{l|}{ TP } & \multicolumn{1}{|c|}{ TN } & FP & FN & TRP & TRN & TEP & TEN & UPP & PPP \\
\hline $1: 9$ & 0.995 & 0.660 & 0.904 & 0.167 & 202 & 322 & 104 & 1 & 35 & 35 & 203 & 426 & 359 & 273 \\
\hline $2: 8$ & 1.000 & 0.718 & 0.975 & 0.123 & 176 & 314 & 69 & 0 & 62 & 78 & 176 & 383 & 861 & 677 \\
\hline $3: 7$ & 1.000 & 0.735 & 0.985 & 0.110 & 150 & 285 & 54 & 0 & 88 & 122 & 150 & 339 & 1390 & 1153 \\
\hline $4: 6$ & 1.000 & 0.718 & 0.991 & 0.105 & 112 & 263 & 44 & 0 & 126 & 154 & 112 & 307 & 2308 & 1889 \\
\hline $5: 5$ & 1.000 & 0.738 & 0.996 & 0.080 & 79 & 242 & 28 & 0 & 159 & 191 & 79 & 270 & 3211 & 2564 \\
\hline $6: 4$ & 1.000 & 0.778 & 0.996 & 0.064 & 63 & 199 & 18 & 0 & 175 & 244 & 63 & 217 & 3666 & 2959 \\
\hline $7: 3$ & 1.000 & 0.786 & 0.996 & 0.057 & 44 & 154 & 12 & 0 & 194 & 295 & 44 & 166 & 4162 & 3371 \\
\hline $8: 2$ & 1.000 & 0.800 & 0.995 & 0.057 & 32 & 100 & 8 & 0 & 206 & 353 & 32 & 108 & 4555 & 3697 \\
\hline $9: 1$ & 1.000 & 0.588 & 0.985 & 0.100 & 10 & 53 & 7 & 0 & 228 & 401 & 10 & 60 & 5286 & 4350 \\
\hline $10: 10^{*}$ & 1.000 & 1.000 & 1.000 & 0.000 & 238 & 461 & 0 & 0 & 238 & 461 & 238 & 461 & 5600 & 4525 \\
\hline
\end{tabular}

B

\begin{tabular}{|r|l|l|l|l|r|r|r|r|r|r|r|r|r|r|}
\hline RA & RE & PR & AU & ER & \multicolumn{1}{l|}{ TP } & \multicolumn{1}{l|}{ TN } & FP & FN & TRP & TRN & TEP & TEN & UPP & PPP \\
\hline $1: 9$ & 0.981 & 0.699 & 0.908 & 0.145 & 202 & 336 & 87 & 4 & 32 & 38 & 206 & 423 & 318 & 219 \\
\hline $2: 8$ & 1.000 & 0.762 & 0.980 & 0.100 & 179 & 324 & 56 & 0 & 59 & 81 & 179 & 380 & 806 & 590 \\
\hline $3: 7$ & 1.000 & 0.789 & 0.989 & 0.084 & 153 & 295 & 41 & 0 & 85 & 125 & 153 & 336 & 1318 & 1040 \\
\hline $4: 6$ & 1.000 & 0.777 & 0.994 & 0.079 & 115 & 271 & 33 & 0 & 123 & 157 & 115 & 304 & 2226 & 1760 \\
\hline $5: 5$ & 1.000 & 0.781 & 0.997 & 0.066 & 82 & 244 & 23 & 0 & 156 & 194 & 82 & 267 & 3120 & 2416 \\
\hline $6: 4$ & 1.000 & 0.835 & 0.998 & 0.046 & 66 & 201 & 13 & 0 & 172 & 247 & 66 & 214 & 3567 & 2798 \\
\hline $7: 3$ & 1.000 & 0.839 & 0.997 & 0.043 & 47 & 154 & 9 & 0 & 191 & 298 & 47 & 163 & 4060 & 3202 \\
\hline $8: 2$ & 1.000 & 0.875 & 0.998 & 0.036 & 35 & 100 & 5 & 0 & 203 & 356 & 35 & 105 & 4451 & 3522 \\
\hline $9: 1$ & 1.000 & 0.765 & 1.000 & 0.057 & 13 & 53 & 4 & 0 & 225 & 404 & 13 & 57 & 5190 & 4153 \\
\hline $10: 10^{*}$ & 1.000 & 1.000 & 1.000 & 0.000 & 238 & 461 & 0 & 0 & 238 & 461 & 238 & 461 & 5578 & 4485 \\
\hline
\end{tabular}

C

\begin{tabular}{|r|l|l|l|l|r|r|r|r|r|r|r|r|r|r|}
\hline RA & RE & PR & AU & ER & TP & TN & FP & FN & TRP & TRN & TEP & TEN & UPP & PPP \\
\hline $1: 9$ & 0.980 & 0.689 & 0.907 & 0.149 & 199 & 336 & 90 & 4 & 32 & 38 & 203 & 426 & 318 & 219 \\
\hline $2: 8$ & 1.000 & 0.749 & 0.980 & 0.106 & 176 & 324 & 59 & 0 & 59 & 81 & 176 & 383 & 806 & 590 \\
\hline $3: 7$ & 1.000 & 0.773 & 0.988 & 0.090 & 150 & 295 & 44 & 0 & 85 & 125 & 150 & 339 & 1318 & 1040 \\
\hline $4: 6$ & 1.000 & 0.757 & 0.992 & 0.086 & 112 & 271 & 36 & 0 & 123 & 157 & 112 & 307 & 2226 & 1760 \\
\hline $5: 5$ & 1.000 & 0.752 & 0.996 & 0.074 & 79 & 244 & 26 & 0 & 156 & 194 & 79 & 270 & 3120 & 2416 \\
\hline $6: 4$ & 1.000 & 0.797 & 0.996 & 0.057 & 63 & 201 & 16 & 0 & 172 & 247 & 63 & 217 & 3567 & 2798 \\
\hline $7: 3$ & 1.000 & 0.786 & 0.994 & 0.057 & 44 & 154 & 12 & 0 & 191 & 298 & 44 & 166 & 4060 & 3202 \\
\hline $8: 2$ & 1.000 & 0.800 & 0.993 & 0.057 & 32 & 100 & 8 & 0 & 203 & 356 & 32 & 108 & 4451 & 3522 \\
\hline $9: 1$ & 1.000 & 0.588 & 0.985 & 0.100 & 10 & 53 & 7 & 0 & 225 & 404 & 10 & 60 & 5190 & 4153 \\
\hline $10: 10^{*}$ & 1.000 & 1.000 & 1.000 & 0.000 & 235 & 464 & 0 & 0 & 235 & 464 & 235 & 464 & 5504 & 4331 \\
\hline
\end{tabular}

D

\begin{tabular}{|c|c|c|c|c|c|c|c|c|c|c|c|c|c|c|}
\hline \multicolumn{5}{|c|}{$\begin{array}{c}\text { Faults in testing } \\
\text { cases 697, 698, and 699) }\end{array}$} & \multicolumn{5}{|c|}{$\begin{array}{c}\text { Faults in training } \\
\text { (cases } 6,13 \text {, and } 15 \text { ) }\end{array}$} & \multicolumn{5}{|c|}{$\begin{array}{c}\text { Faults in training and testing (cases 6, } \\
13,15,697,698 \text {, and 699) }\end{array}$} \\
\hline RA & 0 & NT & PS & NS & RA & 0 & NT & PS & NS & RA & 0 & NT & PS & NS \\
\hline $1: 9$ & 697 & 0 & 42 & 0 & 1:9 & 223 & 0 & 0 & 50 & $1: 9$ & 697 & 0 & 33 & 0 \\
\hline $1: 9$ & 698 & 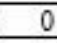 & 9 & 0 & $1: 9$ & 286 & 0 & 0 & 13 & $1: 9$ & 698 & ) & 9 & 0 \\
\hline $1: 9$ & 699 & 0 & 23 & 0 & $1: 9$ & 345 & 7 & & 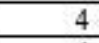 & $1: 9$ & 69 & ) & 10 & 0 \\
\hline & & & & & $1: 9$ & 569 & 0 & & 7 & & & & & \\
\hline $9: 1$ & 697 & 0 & 2672 & 0 & $2: 8$ & 223 & 0 & 4 & 875 & $9: 1$ & 69 & 0 & 2293 & 0 \\
\hline $9: 1$ & 698 & $\underline{0}$ & 134 & 0 & $2: 8$ & 286 & 0 & 123 & 9 & $9: 1$ & 69 & 0 & 134 & 0 \\
\hline $9: 1$ & 699 & 0 & 1016 & 8 & $2: 8$ & 345 & 0 & 12 & 0 & $9: 1$ & 69 & 0 & 1016 & 9 \\
\hline $10: 10^{\prime}$ & 695 & 0 & 0 & 95204 & $2: 8$ & 569 & 0 & 13 & 0 & $10: 10^{\circ}$ & 69 & 0 & 0 & 95204 \\
\hline $10: 10^{\circ}$ & 696 & 0 & 0 & 3537383 & $10: 10^{2}$ & 5 & 0 & 0 & 143716 & $10: 10^{2}$ & 696 & 0 & 0 & 3537383 \\
\hline $10: 10$ & 697 & 0 & 0 & 99 & $10: 10^{\circ}$ & 6 & 0 & 0 & 81 & $10: 10^{\circ}$ & 69 & 0 & 0 & 117 \\
\hline $10: 10^{2}$ & 698 & 0 & 0 & 106 & $10: 10^{2}$ & 13 & 0 & 0 & 726 & $10: 10^{2}$ & 698 & 0 & 0 & 106 \\
\hline $10: 10^{2}$ & 699 & 0 & 0 & 140 & $10: 10^{2}$ & 15 & 0 & 0 & 81 & $10: 10^{\circ}$ & 699 & 0 & 0 & 140 \\
\hline
\end{tabular}

\section{Figure 4}

Tolerance to faulty class labels in BCWO (B). (A) For the case of testing, we change class labels of observations (i.e., 697, 698, and 699) from " 1 " to "0". (B) For the case of training, class labels of observations (i.e., 6, 13, and 15) are changed from "1" to " 0 ". (C) For both cases, we change class labels of observations (i.e., 6, 13, 15, 697, 698, and 699) from "1" to "0". (D) Although faults in testing, TC can still classify $O_{697}, O_{698}$, and $O_{699}$ as PO since the ratio $1: 9$. Regarding faults in training, the change results 
in an additional three errors at the ratio 1:9, i.e., $O_{286}, O_{345}$, and $O_{569}$. Since the ratio $2: 8$, the three errors are eliminated due to increased training data. Although faults in testing and training, TC can still classify $O_{697}, O_{698}$, and $O_{699}$ as $\mathrm{PO}$ since the ratio 1:9. Note at the ratio $10: 10^{\#}, O_{697}, O_{698}$, and $O_{699}$ belong to training data so that their PS are zero; nevertheless, we can identify them by NS. 